\title{
Relationship Between Cardiac Surgery and Acute Ischemic Stroke: An Examination in Terms of Clinical, Radiological, and Functional Outcomes and Possible Pathophysiological Mechanisms
}

\author{
Mehmet Iş1k, ${ }^{1}$ Hasan Hüseyin Kozak, ${ }^{2}$ Niyazi Görmüşs ${ }^{1}$ \\ ${ }^{1}$ Department of Cardiovascular Surgery, Meram Faculty of Medicine, Necmettin Erbakan University, Konya, Turkey; \\ ${ }^{2}$ Department of Neurology, Meram Faculty of Medicine, Necmettin Erbakan University, Konya, Turkey
}

\section{ABSTRACT}

Aim: The aim is to discuss the clinical characteristics, time, anatomical vascular distribution, radiological features, functional outcomes after stroke and possible pathophysiological mechanisms of acute ischemic stroke (AIS) that develop after cardiac surgery.

Method: A total of 3,474 patients, who underwent cardiac surgery between 2015-2020, retrospectively were analyzed. Forty-nine patients, who developed AIS and had brain $\mathrm{CT}$ and diffusion MR images during hospitalization, were included in the study.

Results: AIS distribution was at 53\% CABG, $12.2 \%$ isolated mitral valve, $8.1 \%$ isolated aortic valve, and $26.5 \% \mathrm{com}-$ bined surgical procedures. Patients with a $\leq 2$ days $(P=0.03)$ preop preparation time and body surface area (BSA) of $<1.85$ $\mathrm{m} 2(P=0.02)$ had a high discharge rate. While newly developing $\mathrm{AF}$ was low in the early stroke group, it was higher in the late stroke group $(P=0.02)$. A history of previous cerebrovascular events was found in $3.3 \%$ of the patients. Postoperative new AIS was detected in $7.8 \%$ of those with a history of cerebrovascular events. Total anterior circulation infarction (TACI) case rate was $8.1 \%$, partial anterior circulation infarction (PACI) $12.2 \%$, posterior circulation infarction (POCI) $24.4 \%$, cortical border zone infarction (CBZI) $30.6 \%$, combined POCI + CBZI 12.2\%, multiple territorial infarcts (MTI) 10.2\%, and lacunar circulation infarction (LACI) rate was $2 \%$. The modified Rankin Scale means following AIS was 3.45. The worst Rankin score was 5.75 in $\mathrm{CABG}+\mathrm{MVR}$ cases; it was found to be 5 in the valve + ascending aorta case and 5 in the five bypass cases.

Conclusion: Calculation of cerebrovascular reserve with extra/intracranial vascular imaging is important in patients with multiple risk factors, whose association with stroke has been

Received May 31, 2021; received in revised form fune 12, 2021; accepted fune 16, 2021.

Correspondence: Mehmet Işık, MD, Assistant Professor, Department of Cardiovascular Surgery, Meram Faculty of Medicine, Necmettin Erbakan University, 42080, Konya, Turkey, Telephone +90 5335507549 (e-mail: drmisik@hotmail.com). determined before cardiac surgery. We believe that cardiovascular surgery and neurology multidisciplinary prospective randomized studies should be conducted to obtain pre-, peri- and post-procedural risk calculation scales, according to cardiac surgery type and to reshape surgical procedures accordingly.

\section{INTRODUCTION}

Stroke is a serious complication of cardiac surgery. The shadowing of the success of the surgery causes an increase in hospital costs, morbidity, and mortality. Symptomatic stroke after cardiac surgery is between 1.2-8.4\% [Wolman 1999; Floyd 2006].

There are many etiological reasons that can lead to stroke. Some of the most common risk factors are advanced age, hypertension (HT), diabetes mellitus (DM), hypercholesterolemia, atrial fibrillation (AF), physical inactivity, obesity, genetics, previous stroke history, and smoking. The etiology of ischemic stroke is due to a thrombotic or embolic event that causes a decrease in blood flow to the brain. In a thrombotic event, blood flow to the brain is blocked within the blood vessel, usually due to dysfunction of the vessel itself, secondary to atherosclerotic disease, arterial dissection, or an inflammatory condition. In an embolic event, embolic debris originating from elsewhere in the body blocks blood flow in the affected vessel area. The etiology of stroke affects both prognosis and outcome [Hui 2020; Filsoufi 2008].

Stroke that develops after cardiac surgery has been classified as early and late in order to better understand its characteristics, and efforts have been made to explain it mostly by hypoperfusion and embolization mechanisms [Gaudino 2019; Hedberg 2011].

Stroke clinic provides important insights in understanding and managing the anatomical and radiological distribution of stroke-related lesions, functional outcomes after stroke, possible underlying mechanisms associated with stroke, clinical course, and possible outcomes after stroke [Campbell 2019]. Therefore, it is important to obtain and evaluate these data for strokes after cardiac surgery. This study aims to discuss the clinical characteristics, time, anatomical vascular distribution, radiological characteristics, functional outcomes after stroke, and possible pathophysiological mechanisms of acute ischemic stroke (AIS) developing after cardiac surgery. 


\section{MATERIAL AND METHODS}

Data collection: A total of 3,474 patients, who underwent cardiac surgery between January 2015 and July 2020, retrospectively were analyzed. Study design and case numbers are given in Table 1. (Table 1) Forty-nine patients, who developed postoperative acute cerebrovascular accident (CVA) during hospitalization, were included in the study. By obtaining written informed consent of the surgical patient, the information of the patients who were operated on was obtained from the hospital software system, medical records, and physician follow-up notes. For this retrospective screening, a study protocol approval (2020/2841) was obtained from the local ethics committee of the faculty.

Early stroke was defined as the stroke detected during the extubation phase after the patient regained consciousness. Late stroke was defined as a stroke that developed after an asymptomatic interval after extubation. Cases were classified as coronary artery bypass graft operation (CABG), isolated mitral valve replacement/repair (MVR/R), isolated aortic valve replacement (AVR), and combined procedures (CABG and valve surgeries, multiple valve surgeries, ascending aortic and valve surgeries).

Patients who developed an acute stroke clinic during hospitalization, were examined by a neurologist, and who had brain CT and brain diffusion MR images were included in the study. Cases with neurological examination findings of a stroke clinic but whose radiological imaging could not be performed due to generally patient-related reasons $(N=12)$ and acute stroke cases that developed after discharge were excluded from the study. The demographic data and surgery information of the patients are given in Table 2 in detail. (Table 2)

Surgical method: On-pump CABG was performed by placing a cross-clamp on the aorta and giving cardioplegia. Proximal anastomoses were performed with a side clamp after the heart started beating. Left internal mammarian artery (LIMA) was used in all patients. In off-pump CABG, a side clamp was used for proximal anastomoses of grafts other than LIMA. Off-pump CABG was preferred in cases of calcific or porcelain aorta. Peripheral cannulation was not preferred in such cases, since it is mostly calcified in peripheral arteries. When multiple bypass was required, the sequential technique was used, and proximal anastomoses were made on the LIMA as much as possible. Other surgeries were performed with standard cannulation and CPB (cardiopulmonary bypass) procedures.

Vascular distribution of stroke and functional scoring: Stroke was defined as the presence of rapidly developing focal neurological signs or symptoms of vascular origin. All events were adjudicated by a neurologist. A general medical history was collected and physical and neurological examinations, standard laboratory tests, and 12-lead electrocardiogram were performed on all patients upon admission. In our study, noncontrast brain CT and diffusion-weighted MRIs (dwMRI) were taken into consideration, as well as the clinical findings detected in patients who were evaluated with a suspicion of stroke. Location of the infarct lesion was determined by radiological findings, and the vascular distribution of stroke was classified as follows: A total anterior circulation infarct
(TACI) is a large cortical stroke affecting the areas of the brain supplied by both the middle and anterior cerebral arteries. A partial anterior circulation infarct (PACI) is a less severe form of TACI, in which only part of the anterior circulation has been compromised. A posterior circulation infarct (POCI) involves damage to the area of the brain supplied by the posterior circulation. A lacunar infarct (LACI) is a subcortical stroke that occurs secondary to small vessel disease [Bamford 1991]. Border zones are areas that lie at the junction of two different drainage areas. Border zone infarcts (BZI) are ischemic lesions that occur in characteristic locations at the junction between two main neighboring arterial territories. These territories can be further classified in two broad categories as (a) external (cortical) or (b) internal (subcortical) border zones [Torvik 1984]. Multiple territorial infarcts (MTI) are traditionally defined on neuroimaging as noncontiguous infarcts located in more than one cerebral circulation [Akhtar 2019]. Functional score was evaluated by a trained neurologist using the modified Rankin Scale (mRS). mRS is a commonly used scale for measuring the degree of disability or dependence in the daily activities of people who have suffered a stroke or other causes of neurological disability. The scale runs from 0-6: 0 = No symptoms; 1 = No significant disability, able to carry out all usual activities despite some symptoms; 2 = Slight disability, able to look after own affairs without assistance, but unable to carry out all previous activities; $3=$ Moderate disability, requires some help, but able to walk unassisted; 4 = Moderately severe disability, unable to attend to own bodily needs without assistance, and unable to walk unassisted; $5=$ Severe disability, requires constant nursing care and attention, bedridden, incontinent; 6 = Deceased [Wilson 2002].

Statistical analysis: Statistical analyses were performed using SPSS ver. 21.0 (SPSS Inc. Chicago, Illinois, USA). Patients were grouped according to stroke types (TACI, PACI, POCI, LACI, CBZ, and MTI), stroke times (early/ delayed), and discharge status (discharge/exitus). The difference between categorical parameters was analyzed using the chi-square test. Frequency and percentage values of the parameters were calculated. For all statistical tests $\mathrm{P}<0.05$ was considered statistically significant.

\section{RESULTS}

Demographic and surgery-related results: Of the 49 patients who developed AIS, 17 were female and 32 were male. Mean age was 65.1 (34-81) years, mean aortic clamp time (ACT) was 64.4 minutes, mean body mass index was 28.4, mean body surface area (BSA) was $1.85 \mathrm{~m} 2$, mean discharge time was 16 days, and mean death time was 17 days. It was found that $69.3 \%$ of the cases were discharged and $30.6 \%$ died. Ischemia-related radiological findings were detected in all 49 patients, who were evaluated with a suspicion of stroke and whose cranial radiological examination was performed, while no findings related to hemorrhage were found. AIS distribution was as follows: $26 \mathrm{CABG}$, six isolated $\mathrm{MVR} / \mathrm{R}$, four isolated AVR, and 13 combined surgical procedures. Early stroke was seen in $55.1 \%$ (27) and late stroke in $44.8 \%$ (22) of the 
patients. The number of surgical cases and stroke numbers, according to the type of surgery, are given in Table 1 .

Twenty-nine patients $(59.1 \%)$ in the study group were older than 65 years and $60 \%$ of the patients who died were in this group. In the presence of additional disease that needs examination and treatment in the preoperative preparation phase, it was determined that the preop preparation time was $\geq 3$ days, and the preop preparation time was $\leq 2$ days in other patients. The preop preparation time of $57.1 \%$ (28) of the patients in the study group was $\geq 3$ days, and $86.6 \%(13 / 15)$ of the patients who died were in this group. In cases with a preop preparation time of $\leq 2$ days, the rate of discharge was significantly higher $(P=0.03)$. The discharge rate was significantly higher $(P=0.02)$ in patients with BSA $<1.85 \mathrm{~m}^{2}$. The rate of discharge in patients without DM and with $\mathrm{EF}>50 \%$ was near significantly higher $(P=0.06)$. (Table 3 )

Early stroke was found in $83.3 \%(5 / 6)$ in MVR/R cases, $69.2 \%(9 / 13)$ in combined surgeries, $46.1 \%(12 / 26)$ in CABG, and $25 \%(1 / 4)$ in AVR cases. All patients with chronic AF in the preop period had an early stroke. Newly developing AF was found to be significantly $(P=0.02)$ lower in the early stroke group. Twelve out of 17 patients $(70.5 \%)$ with ACT $>65$ minutes had an early stroke. Nine out of 27 patients $(33.3 \%)$ in the early stroke group died. Four out of 27 patients (14.8\%) in the early stroke group had a history of preoperative stroke.

Fifteen out of 22 patients $(68.1 \%)$ in the late stroke group were over 65 years old. Newly developing AF was significantly $(P=0.02)$ higher in the late stroke group. Six out of
22 patients $(27.2 \%)$ in the late stroke group died. Five out of 22 patients $(22.7 \%)$ in the late stroke group had a history of preoperative stroke (Table 3). There was no significant relationship between both early and late stroke and DM and HT.

Number of patients discharged was $83.3 \%(5 / 6)$ in MVR/R, $75 \%(3 / 4)$ in AVR, $73 \%(19 / 26)$ in CABG, and $53.8 \%(7 / 13)$ in combined surgeries. (Table 4)

Of the 3,381 patients, 114 (3.3\%) had a history of previous CVA. Postoperative AIS was detected in nine $(7.8 \%)$ of these 114 patients. Of the patients with newly detected AIS, eight were CABG patients, and one was an AVR + MVR case. While seven $(77.7 \%)$ of nine patients were discharged, two (22.3\%) CABG patients died.

Neurological results: The TACI case rate was $8.1 \%$, PACI $12.2 \%$, POCI $24.4 \%$, CBZI $30.6 \%$, combined POCI + CBZI $12.2 \%$, MTI $10.2 \%$, and LACI rate was $2 \%$. (Table 5 )

All of the PACI cases were under 65 years old $(\mathrm{N}=6 ; \mathrm{P}=$ $0.003)$. Over the age of 65 were $83.3 \%$ of the isolated POCI cases $(\mathrm{N}=10 ; \mathrm{P}=0.009) ; 60 \%$ of isolated CBZI cases; $83.3 \%$ of the combined POCI + CBZI cases.

Male were $75 \%$ of TACI cases, $83.3 \%$ of PACI cases, $80 \%$ of isolated CBZI cases $(P=0.046)$, and $83.3 \%$ of combined $\mathrm{POCI}+\mathrm{CBZI}$ cases.

HT in $91.6 \%(\mathrm{~N}=11 ; P=0.014)$ of isolated POCI infarction cases and $\mathrm{DM}+\mathrm{HT}$ association was found in $50 \%$ of TACI, PACI, and isolated POCI cases.

It was observed that $100 \%$ of PACI cases, $83.3 \%$ of isolated POCI cases, and $73.3 \%$ of isolated CBZI cases were

Table 1. Study design and stroke rate by type of surgery. CABGO, coronary artery bypass graft operation; MVR/R, mitral valve replacement or repair; AVR, aortic valve replacement; AIS, acute ischemic stroke

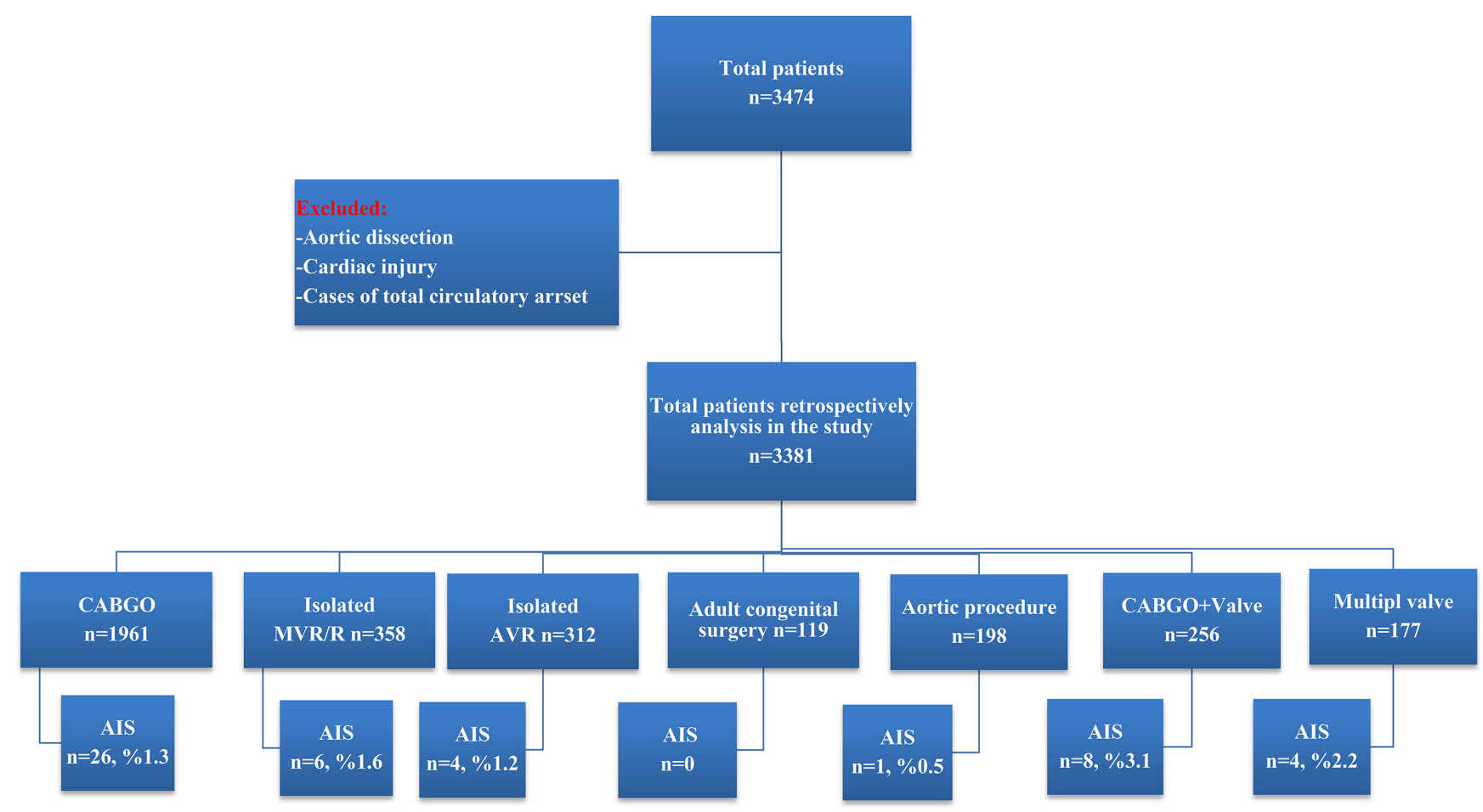


Table 2. Detailed information on demographics of patients and the surgeries performed

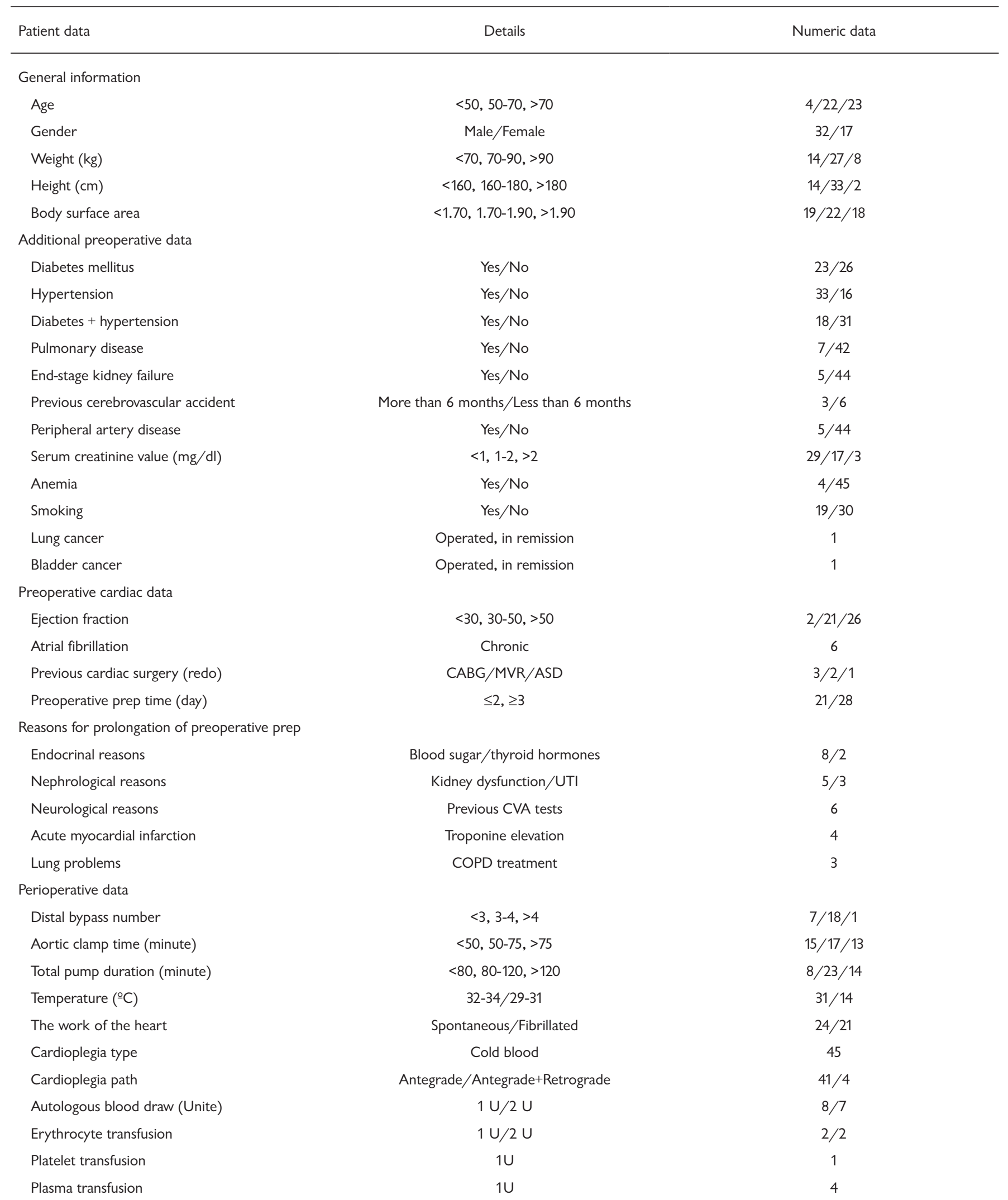




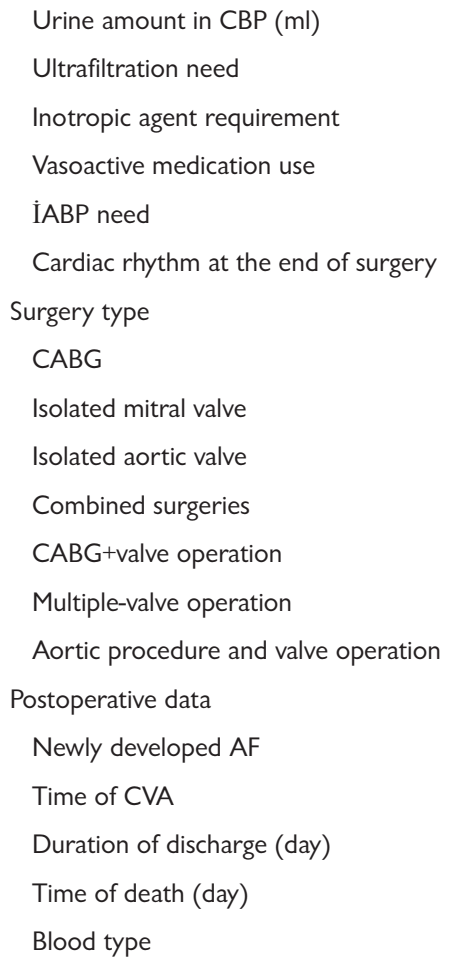

\begin{tabular}{|c|c|}
\hline$<300 />300$ & $27 / 18$ \\
\hline$<300 />300$ & $1 / 3$ \\
\hline Yes/No & $41 / 8$ \\
\hline Yes/No & $2 / 47$ \\
\hline Yes/No & $5 / 44$ \\
\hline Sinus/AF/Pacemaker & $42 / 6 / 1$ \\
\hline On-pump/Off-pump & $22 / 4$ \\
\hline MVR/Mitral repair & $5 / 1$ \\
\hline AVR & 4 \\
\hline Total number & 13 \\
\hline $\mathrm{CABG}+\mathrm{AVR} / \mathrm{CABG}+\mathrm{MVR} / \mathrm{CABG}+\mathrm{TRA}$ & $3 / 4 / 1$ \\
\hline$A V R+M V R / M V R+T V R$ & $3 / 1$ \\
\hline Bentall & 1 \\
\hline First 24 hours/24-48 hours/48-72 hours & $4 / 3 / 1$ \\
\hline First 24 hours/24-72 hours/after 72 hours & $15 / 18 / 16$ \\
\hline$<7,7-14,>14$ & $2 / 15 / 17$ \\
\hline$<7,10-14,>14$ & $1 / 9 / 5$ \\
\hline $\mathrm{A}+/ \mathrm{O}+/ \mathrm{B}+/ \mathrm{AB}+/ \mathrm{A}-/ \mathrm{O}-$ & $19 / 16 / 6 / 3 / 2 / 3$ \\
\hline
\end{tabular}

CABG, coronary artery bypass graft operation; MVR, mitral valve replacement; ASD, atrial septal defect; UTI, urinary tract infection; CVA, cerebrovascular accident; COPD, chronic obstructive pulmonary disease; CPB, cardiopulmonary bypass; IABP, intraaortic balloon pump; AF, atrial fibrillation; AVR, aortic valve replacement; TRA, tricuspid ring annuloplasty; TVR, tricuspid valve replacement

Table 3. Categorical parameters by discharge/death and early/late stroke

\begin{tabular}{|c|c|c|c|c|c|c|c|c|c|c|c|}
\hline Death $(N=15)$ & 9 & 11 & 2 & 1 & 5 & 8 & 8 & 8 & 8 & 13 & 10 \\
\hline Early stroke $(N=27)$ & 14 & 12 & 5 & 6 & 2 & 15 & 10 & 12 & 8 & 14 & 10 \\
\hline
\end{tabular}

DM, diabetes mellitus; CVA, cerebrovascular accident; CAF, chronic atrial fibrillation; NAF, new atrial fibrillation; SS, spontaneous study; SF, study in fibrillation; $\mathrm{ACT}$, aortic clamp time; $\mathrm{EF}$, ejection fraction; BSA, body surface area

discharged. It was found that $75 \%$ of TACI cases and $60 \%$ of MTI cases died.

Early stroke was seen in $75 \%$ of TACI cases and $66.6 \%$ of combined POCI + CBZI cases.

In $75 \%$ of TACI cases, ACT was $>65$ minutes while in $66.6 \%$ of isolated POCI and in isolated CBZI cases, ACT was found to be $<65$ minutes.

BSA was found to be $>1.85$ in $66.6 \%$ of PACI cases, in $100 \%$ of combined POCI + CBZI cases, and in $60 \%$ of MTI cases.

New developing AF was detected in $50 \%$ of the TACI and combined POCI + CBZI cases. While the beating of the heart was spontaneous in $66.6 \%$ of isolated CBZI cases $(N=10 ; P=$ $0.049)$, it was observed that the heart worked by defibrillating in $83.3 \%$ of the PACI cases and $60 \%$ of the MTI cases.

Of the cases with a history of stroke, $44.4 \%$ were isolated POCI and $33.3 \%$ were isolated CBZI.

After AIS, the mean modified Rankin Scale (mRs) was 3.45. While the worst Rankin score was 5.75 in the CABG + MVR cases, it was found to be 5 in the valve + ascending aorta case and 5 in the five bypass cases. (Table 6 )

\section{DISCUSSION}

We discussed the results of the study from two different aspects: surgical and neurological. 
Discussing the surgical results: When the patients in the study group with a preoperative preparation time of $\leq 2$ days were compared with those with $\geq 3$ days, it was observed that there were additional diseases in the group with prolonged periods and that their preoperative examination and treatment took time. Of the patients who had AIS, $57.1 \%$ were in the group whose preoperative preparation time was prolonged. At the same time, $86.6 \%$ of those who died were in

Table 4. Categorical parameters by the type of surgery

\begin{tabular}{lccccccccccc}
\hline Type of surgery & $\begin{array}{c}\text { Early } \\
\text { stroke } \\
(N=27)\end{array}$ & $\begin{array}{c}\text { Late } \\
\text { stroke } \\
(N=22)\end{array}$ & $\begin{array}{c}\text { Discharge } \\
(N=34)\end{array}$ & $\begin{array}{c}\text { Death } \\
(N=15)\end{array}$ & $\begin{array}{c}\text { Preop CVA } \\
(N=9)\end{array}$ & $\begin{array}{c}\text { CAF } \\
(N=6)\end{array}$ & $\begin{array}{c}\text { NAF } \\
(N=8)\end{array}$ & $\begin{array}{c}\text { SS } \\
(N=24)\end{array}$ & $\begin{array}{c}\text { SF } \\
(N=21)\end{array}$ & $\begin{array}{c}\text { ACT }>65 \text { min } \\
(N=17)\end{array}$ & $\begin{array}{c}\text { EF }<50 \% \\
(N=17)\end{array}$ \\
\hline CABGO $(N=26)$ & 12 & 14 & 19 & 7 & 8 & 0 & 3 & 12 & 10 & 3 \\
AVR $(N=4)$ & 1 & 3 & 3 & 1 & 0 & 0 & 0 & 2 & 2 & 0 \\
MVR/R $(N=6)$ & 5 & 1 & 5 & 1 & 0 & 4 & 0 & 2 & 4 & 2 \\
Combined $(N=13)$ & 9 & 4 & 7 & 6 & 1 & 2 & 5 & 8 & 5 & 2 \\
\hline
\end{tabular}

CABGO, coronary artery bypass graft operation; AVR, aortic valve replacement; MVR/R, mitral valve replacement or repair; CVA, cerebrovascular accident; CAF, chronic atrial fibrillation; NAF, new atrial fibrillation; SS, spontaneous study; SF, study in fibrillation; ACT, aortic clamp time; EF, ejection fraction

Table 5. Infarct types and categorical parameters

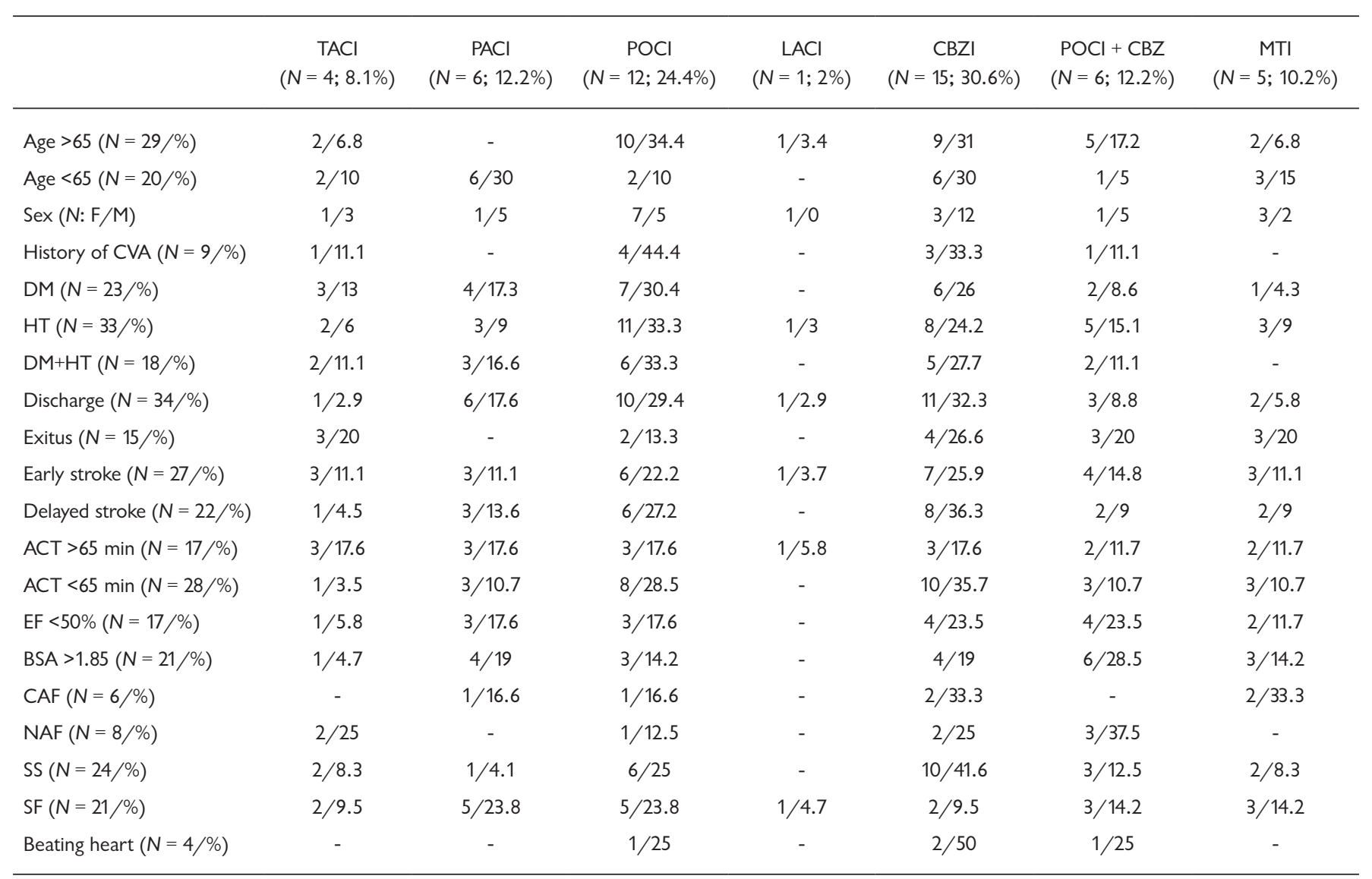

$\mathrm{TACl}$, total anterior circulation infarct; PACl, partial anterior circulation infarct; POCl, posterior circulation infarct; LACl, lacunar infarct; CBZI, cortical border zone infarct; MTI, multiple territorial infarct; Ex, Exitus; PmRs, postoperative modified Rankin scale; CVA, cerebrovascular accident; DM, diabetes mellitus; HT, hypertension; EF, ejection fraction; ACT, aortic clamp time; BSA, body surface area; CAF, chronic atrial fibrillation; NAF, new atrial fibrillation; SS, spontaneous study; SF, study in fibrillation 
this group. The discharge rate $(P=0.03)$ was high in cases with a preop preparation time of $\leq 2$ days. Preoperative preparation times generally are prolonged due to infections, cardiovascular, respiratory, endocrine and nephrological reasons, and perioperative and/or postoperative complications are frequently encountered in patients with these additional problems. It is possible that study data are associated with adverse outcomes and even stroke in patients with prolonged preoperative preparation.

We think that the type of surgery is associated with the AIS development mechanism. Especially in patients who underwent MVR due to mitral stenosis, calcific ulcerated lesions in commissures or vegetations in the valve may fall into the ventricle while the valve is removed (In such cases, it may be beneficial to start removing the valve from the non-calcified side in order to reduce the possibility of embolism). Detecting these particles that may cause embolism is more difficult in MVR cases than in AVR cases, due to the angle of view. Strokes that may occur in cases of MVR may be associated with this condition. In our study, early stroke was found with a high rate $(83.3 \%)$ in MVR/R cases. If we associate early stroke with surgicalinduced particle embolization, it can be said that one of the possible causes that may lead to stroke is the type of surgery.

Early stroke also was observed more frequently $(69.2 \%)$ in combined cases. Possible reason for the prevalence of early stroke in these cases may be related to the long ACT and the MVR in one of the combined operations in five patients. In CABG, early and late stroke was almost half. Late stroke (75\%) was more common in AVR cases. Although the small number of cases $(N=4)$ makes it difficult to comment on late stroke, the mean age of AVR cases being five years higher than the mean age of the study group and the fact that new $\mathrm{AF}$ was not detected in these cases may constitute the data for possible AVR-related stroke pathophysiology mechanism studies.

It has been reported that there are risks associated with atherosclerosis and cardiopulmonary bypass in the background of early stroke [Hedberg 2011; Tarakji 2011]. Embolism resulting from the rupture and displacement of atherosclerotic plaque, thrombus, or fat particles due to manipulation of the aorta and heart may cause early stroke. During CPB, micro embolisms due to air, gas or particles can be seen [Tarakji 2011]. The sources of air and gas embolism are the connection points, air in the lines, loose fit of the purse suture used for cannulation, and rapid heating of cold blood in the pump. Since the extracorporeal circulation is not entered in off-pump CABG, the risks of embolism arising from CPB are eliminated. This situation can be considered as an advantage over on-pump CABG in terms of embolism sources.

It also has been reported that patients with a history of carotid or cerebral artery atherosclerosis may develop

Table 6. Infarct types and Modified Rankin Scoring by surgery types

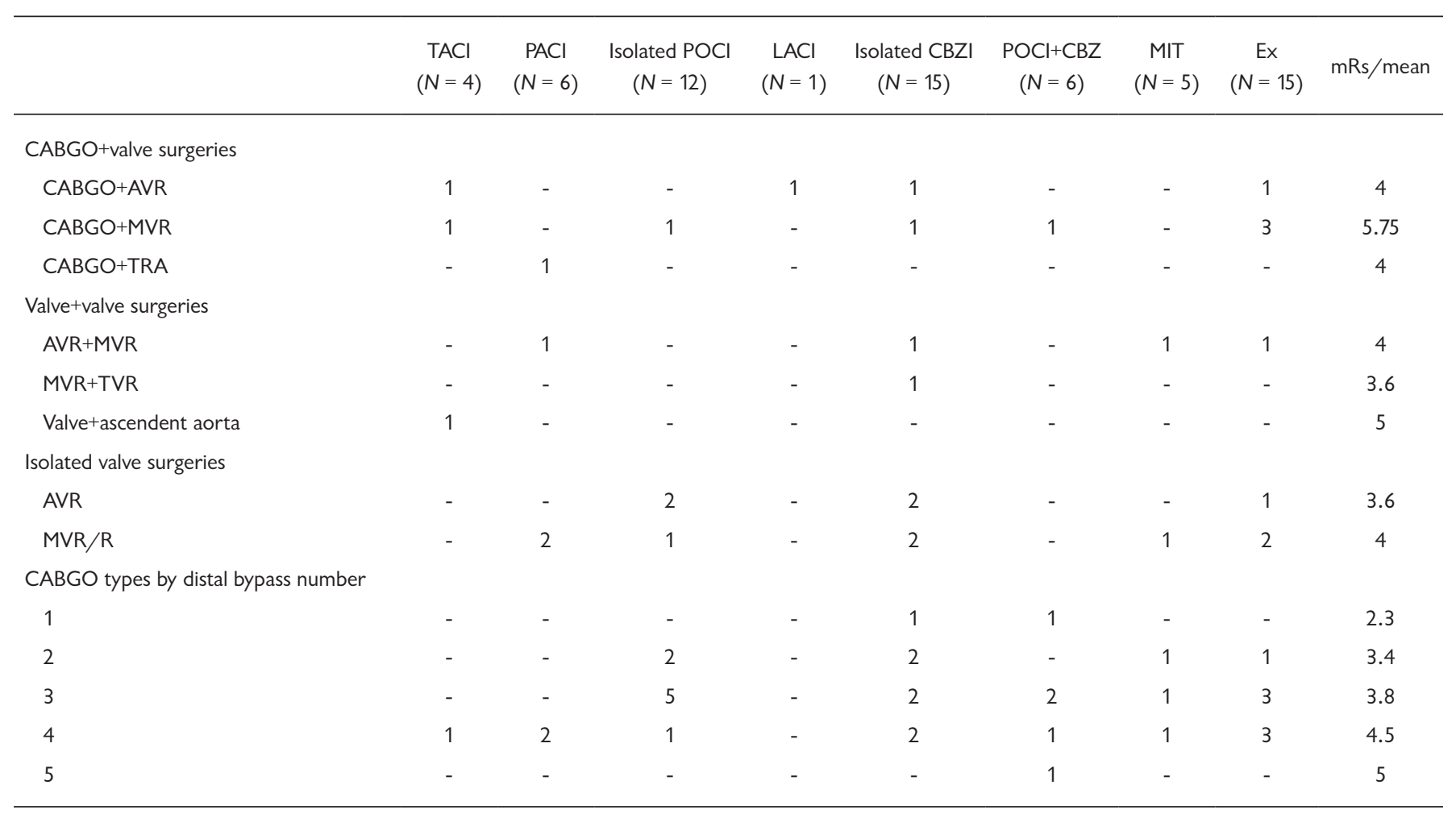

$\mathrm{TACl}$, total anterior circulation infarct; $\mathrm{PACl}$, partial anterior circulation infarct; $\mathrm{POCl}$, posterior circulation infarct; LACI, lacunar infarct; CBZI, cortical border zone infarct; MTI, multiple territorial infarct; Ex, Exitus; mRs, modified Rankin scale; CABGO, coronary bypass graft operation; AVR, aortic valve replacement; $M V R / R$, mitral valve replacement or repair; TRA, tricuspid ring annuloplasty; TVR, tricuspid valve replacement 
premature stroke as a result of intraoperative hypoperfusion [Lee 2011]. We did not have a preoperative pre-examination of the cerebrovascular reserve and atherosclerotic load of the aorta of the patients in our study group. In addition, early stroke was found in $70.5 \%$ of patients with ACT $>65$ minutes. During aortic clamp blood flow is nonpulsatile and arterial blood pressure values remain below normal physiological values. Prolongation of this period may also be associated with early stroke.

It has been reported that late stroke may be associated with atherosclerotic load, perioperative cardiac events, advanced age, postoperative AF, and low cardiac output [Hedberg 2011; Tarakji 2011]. Of the late stroke patients, $68.1 \%$ were over 65 years of age and $75 \%$ of those with postoperative, newly developing $\mathrm{AF}$ had a late stroke. All newly developed AF occurred after patients were extubated and before they had AIS. All of the patients with chronic AF had an early stroke, and none had a late stroke. Left atrial appendix was closed during surgery in patients with chronic AF. In addition, all patients with chronic AF were patients who underwent valve replacement, so low molecular weight heparin/warfarin was given in the early postoperative period. These two conditions may have prevented patients with chronic AF from having a late stroke.

It has been reported that the impact of early stroke on operative mortality is significantly higher than late stroke [Gaudino 2019]. We found mortality rates as $33.3 \%$ and $27.2 \%$ in the early stroke and late stroke groups, respectively.

It has been reported that a history of past CVA is associated with postoperative newly developing CVA and negatively affects survival [Gaudino 2019; Boivie 2005]. Of the 3,381 patients examined, 114 (3.3\%) had a past CVA history. Nine $(7.8 \%)$ of these 114 patients had AIS again postoperatively. Considering all patients, the rate of AIS was 1.44\% $(49 / 3,381)$, while this rate increased to $7.8 \%$ in those with a preoperative CVA history. The discharge rate of the patients in our study group was $67.5 \%(27 / 40)$ in those without a preoperative CVA history, while it was $77.7 \%$ (2/9) in those with a history of preoperative CVA. We are of the opinion that the difference in survival may be related to the type of CVA, how long before the surgery it occurred, the width of the infarct area, and the functional status caused in the patient.

Discussion of neurological results: Of the study group, $59.1 \%$ were patients over the age of 65 . Aging is the most robust non-modifiable risk factor for incident stroke, which doubles every 10 years after age 55. Approximately threequarters of all strokes occur in persons aged $\geq 65$ years [Yousufuddin 2019]. In addition, studies have found a relationship between early or late stroke after cardiac surgery and advanced age [Wolman 1999]. With aging, both cerebral micro and macro-circulations undergo structural and functional alterations. Age-related microcirculatory changes are presumably mediated by endothelial dysfunction and impaired cerebral autoregulation and neurovascular coupling. Endothelial dysfunction promotes neuro-inflammation, impaired cerebral autoregulation may lead to microvascular injury [Gaudino 2019]. In the study group, $60 \%$ of isolated CBZI (30.6\%) cases which is the most frequently detected infarct type and
$83.3 \%(P=0.009)$ of isolated POCI $(24.4 \%)$ cases were over the age of 65 . It was stated in a study that although age is one of the well-known unchangeable stroke risk factors, the age of the patients is not a risk factor for any border zone infarct type [Yong 2006]. However, in a study by The New England Medical Center Posterior Circulation Registry (NEMC-PCR) it was found that the majority of POCI patients were between the ages of 66-75 [Glass 2002]. Border zone infarcts constitute approximately $10 \%$ of all cerebral infarcts. Border zone infarction may be better explained by invoking a combination of two often interrelated processes: hypoperfusion and embolization. Hypoperfusion, or decreased blood flow, is likely to impede the clearance (washout) of emboli. Because perfusion is most likely to be impaired in border zone regions, clearance of emboli will be most impaired in these regions of least blood flow. Also, stenotic disease of the internal carotid artery causes both embolization and decreased perfusion. Similarly, cardiac disease often is associated with micro embolization from the heart and aorta with periods of diminished systemic and brain perfusion. Isolated cortical border zone infarcts may be embolic in nature and are less frequently associated with hemodynamic compromise. Micro emboli from the heart or atherosclerotic plaques in major arteries may preferentially propagate to cortical border zones, which have lower perfusion than other areas of the vasculature and, thus, a limited ability to wash out these emboli [Mangla 2011]. Posterior circulation strokes account for approximately $20-25 \%$ of all strokes, with high mortality and morbidity [Sparaco 2019]. It is stated that approximately $24 \%$ of posterior circulation infarctions are associated with cardiac originated emboli, while $32-35 \%$ are associated with atherosclerotic large vessel disease. In addition, the most common extracranial site of this atherosclerotic disease is the V1 segment of the vertebral artery (VA), that atherosclerotic plaques can start from the subclavian artery and extend to the ostium of the extracranial VA, or that the extracranial Vas can start from the most proximal site, that the severity of posterior circulation strokes due to extracranial VA atherosclerotic disease is related to plaque features, such as the surface irregularity and morphology of the lesion [Al-Ali 2011].

Even if there is no additional cardiovascular morbid condition, the micro/macro structural changes occurring in the extra/intracranial cerebral vascularity that change with aging can disrupt the cerebral blood flow. Moreover, the prevalence of silent cerebrovascular ischemic diseases, transient ischemic attack histories, and minor stroke events increase with advanced age. When additional cardiovascular diseases, which cause impairment in the flow of the cerebrovascular bed and whose effects have been proven, are added to the flow of the event, changes in cerebral vascularity become more pronounced and can be determined by laboratory tests. In fact, with increasing age, cardiovascular diseases significantly increase both primary and secondary negative functional consequences and mortality associated with stroke. Among the results obtained in our study are that the cardiovascular disease load [additional diseases that cause micro/macro structural changes in the cerebrovascular bed such as DM or $\mathrm{HT}$ or DM+HT $(91.6 \%$ of the isolated POCI cases had HT 
$(P=0.014), \mathrm{AF}$ (in all of the patients with chronic AF during the preoperative period, early period stroke was detected), previous stroke history $(33.3 \%$ of the isolated CBZI cases, $44.4 \%$ of the isolated POCI cases, and the entire study group had an AIS rate of $1.44 \%$ (49/3381) while this rate was $7.8 \%$ in those with preoperative stroke history)] directly affecting potential stroke mechanisms (embolization and/or hypoperfusion) prolong the preoperative prep time, increase the risk of stroke and mortality $(57.1 \%$ of the cases had a preoperative preparation time of $\geq 3$ and $86.6 \%$ of the patients who died are in this group) and additionally, the perioperative surgical procedure related risks [the increasing vascular atherosclerotic load with advanced age and additional vascular diseases, prolonged operation time (in $70.5 \%$ of the cases with ACT $>65$, early stroke)] contribute significantly to developing a stroke.

Consequently, preoperative low cardiovascular load reduces the risk of perioperative and postoperative stroke and even increases discharge rates in patients who develop AIS. When we evaluate the problems of advanced age and additional cardiovascular risk loads in the context of the abovementioned information in terms of the vascular mechanisms of isolated CBZI and isolated POCI which were detected frequently in the study group, we conclude that one of the prerequisites for predicting the perioperative stroke risks of cardiac surgeries is to routinely evaluate the extra/intracranial cerebral vascularity with cerebrovascular imaging methods in terms of atherosclerotic, atherothromboembolic and possible conditions that may directly or indirectly cause hypoperfusion. As such, neurovascular imaging is essential to diagnose to improve patient outcomes, determine early and late prognosis, understand the pathogenesis of the event, guide pathogenesis-specific stroke prevention treatments, and identify patients eligible for ongoing clinical trials [AYX 2018]. Also, $46.6 \%$ of the isolated CBZI, $66.6 \%$ of the isolated POCI after CABG operations; $53.3 \%$ of isolated CBZI and $33.3 \%$ of the isolated POCI were after combined operations with valve. These results, in addition to the above suggestions, provide preliminary information that another condition for predicting the perioperative stroke risk is that the surgical operation procedures to be performed should be arranged according to the common stroke types. As a result, as stroke after cardiac surgery is the most unwanted complication by surgeons, it suggests that more professional approaches are needed in cardiovascular surgery planning.

Ischemic strokes occurring in the anterior circulation are the most common of all ischemic strokes, accounting for approximately $70 \%$ of all cases. They are caused most commonly by occlusion of one of the major intracranial arteries or of the small single perforator (penetrator) arteries. The most common causes of arterial occlusion involving the major cerebral arteries are emboli, most commonly arising from atherosclerotic arterial narrowing at the bifurcation of the common carotid artery, from cardiac sources, or from atheroma in the aortic arch and a combination of atherosclerotic stenosis and superimposed thrombosis. The most common sites of occlusion of the internal carotid artery are the proximal $2 \mathrm{~cm}$ of the origin of the artery and, intracranially, the carotid siphon. Factors that modify the extent of infarction include the speed of occlusion and systemic blood pressure. The middle cerebral artery (MCA) is the largest of the intracerebral vessels and supplies through its pial branches. Occlusion of the MCA or its branches is the most common type of anterior circulation infarct, accounting for approximately $90 \%$ of infarcts and two-thirds of all first strokes [Kumral 2002; Nogles 2020]. In our study, the rate of patients with anterior circulation infarction (ACI) was $20.4 \%$. This rate is lower than classical stroke vascular distribution area rates. Also, all of the PACI cases were under 65 years old $(P=0.003)$. Seventy-five percent of TACI and $66 \%$ of PACI were detected after combined operations with valve, and in $50 \%$ of TACI cases postoperative newly developed AF was detected. These findings are at higher rates than classical stroke epidemiology. Considering the results that may be mostly related to surgery, it was found that $75 \%$ of the TACI cases had early stroke, and $75 \%$ had ACT $>65$ minutes. When the current literature information and the findings of our study are combined and the possible mechanism for ACI that develops after cardiac surgery is evaluated, it is concluded that the newly emerged stroke can occur with cardiovascular embolization that is not associated with new embolization or atherosclerosis added to the current atherosclerotic process $(\mathrm{DM}+\mathrm{HT}$ association was detected in $50 \%$ of the ACI cases) and possibly associated with periprocedural surgical load $(75 \%$ of the TACI cases and $66.6 \%$ of the PACI cases were surgeries combined with valve).

Combined POCI + CBZI was detected in $12.2 \%$ of the cases. Of the combined POCI + CBZI cases, $83.3 \%$ were over the age of 65 . Also, $83.3 \%$ of the cases were detected after CABG operations. HT was detected in $83.3 \%$ of the cases, early stroke in $66.6 \%, \mathrm{EF}<50$ in $66.6 \%$, and newly emerged $\mathrm{AF}$ in $50 \%$. Combined POCI + CBZI is rare in routine stroke practice. Both embolization and hypoperfusion are possible mechanisms for both vascular area strokes. In perioperative transcranial Doppler echography studies, embolization to the cerebrovascular bed was found in most patients, and it was found that this situation revealed radiologically more than one infarct type and clinic [Barbut 1996; Barbut 1998]. The combined POCI + CBZI study which revealed advanced age, high vascular comorbidity load and postoperative high rate of newly emerging atrial fibrillation, radiologically confirmed infarct findings in more than one vascular bed leads to the opinion that the possible primary mechanism is more in favor of embolization. At this point, it is obvious that the preoperative condition and reserve of the cerebrovascular bed should be evaluated radiologically in order to consolidate this opinion, and unfortunately, neither in the literature nor in our possession are there sufficient radiological vascular reserve data.

Another type of infarct that can develop perioperatively is MTI. Sixty percent of our MTI cases were detected after CABG operations, $40 \%$ after combined operations with valve, and HT was detected in $60 \%$. Although the classically recommended and/or known mechanism for MTI is cardioembolism, it is important to review the atherosclerotic diseases of extra/intracranial vessels, especially the aorta [Akhtar 
2019; Novotny 2017]. Current study findings also definitely support this data.

The mean modified Rankin scale (mRs) after stroke was 3.45 for all cases. The worst Rankin score was 5.75 in the CABG + MVR cases, while it was 5 in the valve + ascending aorta cases and 5 in the five bypass cases. This result means at best moderate disability, the inability to meet one's own physical needs and to walk unaided, while the worst severe disability means being bedridden requiring constant care or death. Poor functional outcomes overshadow the surgical success, cause more difficult management of the postoperative surgical process, and lead to long hospitalization times, difficult recovery, and mortality in living patients. Considering that combined, complicated, and long operation times bring along stroke, we believe that our postoperative stroke functional outcome data will provide another stimulating point of view to cardiac surgeons for regulations regarding preoperative and postoperative stroke practice.

It was found that $69.3 \%$ of the cases were discharged and $30.6 \%$ died. It was seen that death occurred in $75 \%$ of TACI and $60 \%$ of MTI. It was determined that $100 \%$ of PACI cases, $83.3 \%$ of isolated POCI cases, and $73.3 \%$ of isolated CBZI cases were discharged. Clinical consequences of ischemic brain lesions depend on parenchymal prominence and lesion enlargement. Ischemic lesions range from clinically silent lesions to life-threatening infarctions, and the associated morbidity and mortality varies widely as a result. Largevolume or multiple-site stroke results in worse functional outcome and higher mortality than isolated and small-volume strokes [Murray 1997; Moulin 2000], and the study results indicate this.

It is necessary to specify some factors that limit the study. First, it was a retrospective study. Second, patients who had neurological examination findings of a stroke clinic but who could not have radiological imaging $(N=12)$ due to generally patient-related reasons were excluded from the study. Third, and perhaps most importantly, the lack of information on existing aortic, carotid, or cerebral artery reserves, as preoperative intra/extra cranial vascular examination was not/could not be performed, especially in cases with previous CVA.

As a result, we believe that it is necessary to conduct multidisciplinary prospective randomized studies of cardiac vascular surgery and neurology in order to calculate cerebrovascular reserve with extra/intracranial vascular imaging in patients with high cardiovascular load and multiple risk factors, especially those associated with stroke before cardiac surgery, to develop pre-, peri- and post-procedural risk calculation scales for operations to be performed in the light of literature data and to reshape surgical procedures accordingly, to determine the possible mechanisms of peri and postoperative stroke and determine pre, peri and postoperative treatment modalities.

ACKNOWLEDGEMENT

We thank Associate Professor Yıldırım Kayacan for his contributions in the statistical analysis.
REFERENCES

Akhtar T, Shahjouei S, Zand R. 2019. Etiologies of simultaneous cerebral infarcts in multiple arterial territories: A simple literature-based pooled analysis. Neurol India. 67(3): 692-695.

Al-Ali F, Barrow T, Duan L, et al. 2011. Vertebral artery ostium atherosclerotic plaque as a potential source of posterior circulation ischemic stroke: result from borgess medical center vertebral artery ostium stenting registry. Stroke. 42(9): 2544-2549.

AYX, Coutts SB. 2018. Role of Brain and Vessel Imaging for the Evaluation of Transient Ischemic Attack and Minor Stroke. Stroke. 49(7): 1791-1795.

Bamford J, Sandercock P, Dennis M, Burn J, Warlow C. 1991. Classification and natural history of clinically identifiable subtypes of cerebral infarction. Lancet. 337(8756): 1521-1526.

Barbut D, Grassineau D, Lis E, Heier L, Hartman GS, Isom OW. 1998. Posterior distribution of infarcts in strokes related to cardiac operations. Ann Thorac Surg. 65(6): 1656-1659.

Barbut D, Yao FS, Hager DN, et al. 1996. Comparison of transcranial Doppler ultrasonography and transesophageal echocardiography to monitor emboli during coronary artery bypass surgery. Stroke. 27: 87-90.

Campbell BCV, De Silva DA, Macleod MR, Coutts SB, Schwamm LH, Davis SM, et al. 2019. Ischaemic stroke. Nat Rev Dis Primers. Oct 10; 5(1): 70 .

Filsoufi F, Rahmanian PB, Castillo JG, Bronster D, Adams DH. 2008. Incidence, topography, predictors and long-term survival after stroke in patients undergoing coronary artery bypass grafting. Ann Thorac Surg. 85: 862-71.

Floyd TF, Shah PN, Price CC, et al. 2006. Clinically silent cerebral ischemic events after cardiac surgery: their incidence, regional vascular occurrence, and procedural dependence. Ann Thorac Surg. 81: 2160-6.

Gaudino M, Rahouma M, Di Mauro M, Yanagawa B, Abouarab A, et al. 2019. Versus Delayed Stroke After Cardiac Surgery: A Systematic Review and Meta-Analysis. J Am Heart Assoc. 7(13): e012447.

Glass TA, Hennessey PM, Pazdera L, et al. 2002. Outcome at 30 days in the New England Medical Center Posterior Circulation Registry. Arch Neurol. 59(3): 369-376.

Hedberg M, Boivie P, Engström KG. 2011. Early and delayed stroke after coronary surgery an analysis of risk factors and the impact on shortand long-term survival. Eur J Cardiothorac Surg. 40: 379-8.

Hui C, Tadi P, Patti L. Ischemic Stroke. 2020. 2020 Aug 10. In: StatPearls [Internet]. Treasure Island (FL): StatPearls Publishing; Jan-. PMID: 29763173.

Kumral E, Bayulkem G, Evyapan D, Yunten N. 2002. Spectrum of anterior cerebral artery territory infarction: clinical and MRI findings. Eur J Neurol. 9(6): 615-624.

Lee EJ, Choi KH, Ryu JS, Jeon SB, Lee SW, Park SW, et al. 2011. Stroke risk after coronary artery bypass graft surgery and extent of cerebral artery atherosclerosis. J Am Coll Cardiol. 57: 1811e8.

Mangla R, Kolar B, Almast J, Ekholm SE. 2011. Border zone infarcts: pathophysiologic and imaging characteristics. Radiographics. 31(5): 1201-1214.

Moulin T, Tatu L, Vuillier F, Berger E, Chavot D, Rumbach L. 2000. Role of a stroke data bank in evaluating cerebral infarction subtypes: patterns and outcome of 1,776 consecutive patients from the Besançon stroke registry. Cerebrovasc Dis. 10(4): 261-271. 
Murray CJ, Lopez AD. 1997. Global mortality, disability, and the contribution of risk factors: Global Burden of Disease Study. Lancet. 349(9063): 1436-1442.

Nogles TE, Galuska MA. 2020. Middle Cerebral Artery Stroke. In: StatPearls. Treasure Island (FL): StatPearls Publishing; August 13.

Novotny V, Thomassen L, Waje-Andreassen U, Naess H. 2017. Acute cerebral infarcts in multiple arterial territories associated with cardioembolism. Acta Neurol Scand. 135(3): 346-351.

Sparaco M, Ciolli L, Zini A. 2019. Posterior circulation ischaemic strokea review part I: anatomy, aetiology and clinical presentations. Neurol Sci. 40(10): 1995-2006.

Tarakji KG, Sabik 3rd JF, Bhudia SK, Batizy LH, Blackstone EH. 2011. Temporal onset, risk factors, and outcomes associated with stroke after coronary artery bypass grafting. JAMA. Jan 26; 305(4): 381-90.
Torvik A. 1984. The pathogenesis of watershed infarcts in the brain. Stroke. 15(2): 221-223.

Wilson JT, Hareendran A, Grant M, et al. 2002. Improving the assessment of outcomes in stroke: use of a structured interview to assign grades on the modified Rankin Scale. Stroke. 33(9): 2243-2246.

Wolman RL, Nussmeier NA, Aggarwal A, Kanchuger MS, Roach GW, et al. 1999. Cerebral injury after cardiac surgery: identification of a group at extraordinary risk. Multicenter Study of Perioperative Ischemia Research Group (McSPI) and the Ischemia Research Education Foundation (IREF) Investigators. Stroke. 30: 514-22.

Yong SW, Bang OY, Lee PH, Li WY. 2006. Internal and cortical borderzone infarction: clinical and diffusion-weighted imaging features. Stroke. 37(3): 841-846.

Yousufuddin M, Young N. 2019. Aging and ischemic stroke. Aging (Albany NY). May 1; 11(9): 2542-2544. 